\title{
Successful treatment with long-acting injectable aripiprazole monohydrate for two patients with dual diagnosis - substance use disorder and psychotic disorder
}

\author{
Ali Erdogan ${ }^{1 \oplus}$, Burak Kulaksizoglu $^{1 \oplus}$, Mert Sinan Bingol ${ }^{1 \oplus}$, Buket Cinemre $^{1 \oplus}$, Mehmet Murat Kuloglu $^{\oplus}$ \\ 'Akdeniz University, Faculty of Medicine, Department of Psychiatry, Antalya - Turkey
}

\begin{abstract}
In recent years, the rate of substance-induced psychotic disorder (SIPD) has been gradually increasing. Non-compliance with treatment and substance craving are serious problems in SIPD. Novel depot antipsychotic treatments, such as long-acting injectable aripiprazole monohydrate $400 \mathrm{mg}$ (LAIAM) are reported to improve treatment compliance and functionality in psychotic disorders. However, the number of studies showing the efficacy of LAIAM treatment in patients with dual diagnosis is limited. In this paper, we present two cases. The first case was diagnosed with SIPD and the other was diagnosed with psychotic disorder with a past history of profound substance use disorder. The two cases showed both clinical and functional improvement at 1-year follow-up after the initiation of LAIAM treatment. These case reports suggest that LAIAM treatment might be an effective and safe treatment option in SIPD and reduce substance craving.
\end{abstract}

Keywords: Craving, long-acting injectable aripiprazole, substance-induced psychotic disorder, treatment

\section{INTRODUCTION}

Addictive substances are psychoactive substances that either stimulate or suppress the central nervous system. They impair perception, mood, mental state, behavior, and motor functions. Substance use is a significant factor in the etiology of psychotic disorders $(1,2)$. The rate of psychiatric disorders in people with psychoactive substance use disorder is 3.7 times higher than in the general population, and the rate of at least one comorbid psychiatric disorder among these patients increases up to $\% 90$ (3). Also, there is a distinct diagnostic category for substance-induced psychosis (SIPD) in the Diagnostic and Statistical Manual of Mental Disorders 5
(DSM-5), which involves the presence of delusions and hallucinations (either or both). These symptoms are also required to have started during or shortly after the substance intake or withdrawal. Thus, there should be findings of substance use in medical history, physical examination, or laboratory findings, and this disorder should not be explained by any other etiology (4). Distinguishing between primary psychotic disorder with concurrent substance abuse and SIPD can be diagnostically challenging. Indeed, a systematic review has revealed that there is not much difference in the psychopathology of these disorders. However, a weaker family history of psychotic disorder, more insight, fewer positive symptoms, and fewer negative symptoms, more

How to cite this article: Erdogan A, Kulaksizoglu B, Bingol MS, Cinemre B, Kuloglu MM. Successful treatment with long-acting injectable aripiprazole monohydrate for two patients with dual diagnosis - substance use disorder and psychotic disorder. Dusunen Adam The Journal of Psychiatry and Neurological Sciences 2021;34:102-106.

Correspondence: Ali Erdogan, Akdeniz University, Faculty of Medicine, Department of Psychiatry, Antalya - Turkey 
depression, and more anxiety were reported in SIPD patients in this review (5).

Non-compliance with treatment is relatively higher in psychotic disorders compared to other diseases (6). Comorbid substance use disorder reduces compliance in patients with a psychotic disorder, resulting in poor treatment response and increased recurrence rate. For this reason, antipsychotic drug treatments in intramuscular (i.m.) depot form seems to be promising in the management of cases with comorbid substance abuse and psychotic disorders (7). Long-acting injectable aripiprazole monohydrate $400 \mathrm{mg}$ (LAIAM) is a novel second-generation antipsychotic administered once a month intramuscularly. The LAIAM seems to be comparable to oral aripiprazole regarding efficacy, safety, and tolerability (8).

In this article, we report two SIPD patients who had a history of multiple substance use and showed significant improvement with LAIAM treatment in a 1-year follow-up period.

\section{CASE 1}

The first case is a 31-year-old male married patient with no children. He is a high school graduate and has been unemployed for six months. He was taken to the emergency room by his family, who reported that he had fears of being followed by some malicious people the past few months and would often acted aggressively towards others. He thought the genie was controlling him and heard voices telling him what to do. He had a 15-yearhistory of nicotine use with one pack of cigarettes a day and a 12-year-history of alcohol use once or twice a month. The patient has been also using multiple substances for the last ten years. He said he had started using cannabinoid ten years ago and had been taking it almost every day since then. For the last three years, he has been using skunk several times a week, and he used methamphetamine for a couple of months, almost every day. Skunk is a synthetic cannabis derivative that increases the delta-9 tetrahydrocannabinol level, that provides the psychoactive effect. He had last used methamphetamine a month ago and marijuana and skunk ten days ago. He had no other medical disease and no family history of psychiatric disorders.

There was nothing remarkable about his overall appearance except a giant tattoo on his forearm. He did not have any formal thought disorders, but he was preoccupied with persecutory delusions and had auditory hallucinations commanding him to do something. He said he had suicidal thoughts recently.
His psychomotor activity increased, and despite these severe symptoms he denied that he was ill.

The patient's psychotic symptoms started after substance use, and he had no family history of schizophrenia. Thus he was diagnosed with SIPD according to the DSM-5 diagnostic criteria. The patient was hospitalized, and received $50 \mathrm{mg}$ i.m zuclopenthixol injection daily in the first three days of his admission because he was very agitated during the first days, in addition to the drug regimen including diazepam 30 $\mathrm{mg}$ /day, olanzapine $20 \mathrm{mg}$ /day, carbamazepine $200 \mathrm{mg} /$ day, which had been started ten days ago in another outpatient clinic. Due to high levels of transaminase enzymes in routine biochemistry tests (Alanine aminotransferase - ALT $=111 \mathrm{mg} / \mathrm{dL}$ and aspartate aminotransferase - AST $=58 \mathrm{mg} / \mathrm{dL}$ ) and a gradual increase in follow-up measurements, diazepam, olanzapine, and carbamazepine were discontinued. Later, $20 \mathrm{mg}$ of oral aripiprazole was administered, and additionally, LAIAM was started due to the poor compliance of the patient with oral medications in the past.

The patient was discharged with partial remission after a two-week hospital stay and went on regular follow-up visits once a month at which he received his injection. At one of these visits, the patient admitted that he stopped taking all oral medications soon after his discharge, but agreed to continue LAIAM treatment. At the end of 1-year follow-up with LAIAM treatment alone, almost all of his psychotic symptoms were resolved, and he was clean in terms of substance, enabling him to return his normal functioning (Table 1). During the follow-up, it was confirmed by urine toxicology that the patient did not use any substance. Furthermore, a significant recovery was observed in the patient's liver function test (LFT) values (ALT $=30 \mathrm{mg}$ / $\mathrm{dL}, \mathrm{AST}=17 \mathrm{mg} / \mathrm{dL}$ ) in the third month. No serious side effect was observed in the meantime. The patient is currently included in an addiction program.

\section{CASE 2}

The second case is a 27-year-old single male patient. $\mathrm{He}$ is a high-school graduate and currently works in a supermarket. The patient was diagnosed with a psychotic disorder specified with a past history of profound substance (cannabis, synthetic cannabinoids and ecstasy) use disorder. At such times, he became aggressive and felt suicidal. The patient was restless most of the time, but did not show any physical aggression, and his suicidal tendency never went 


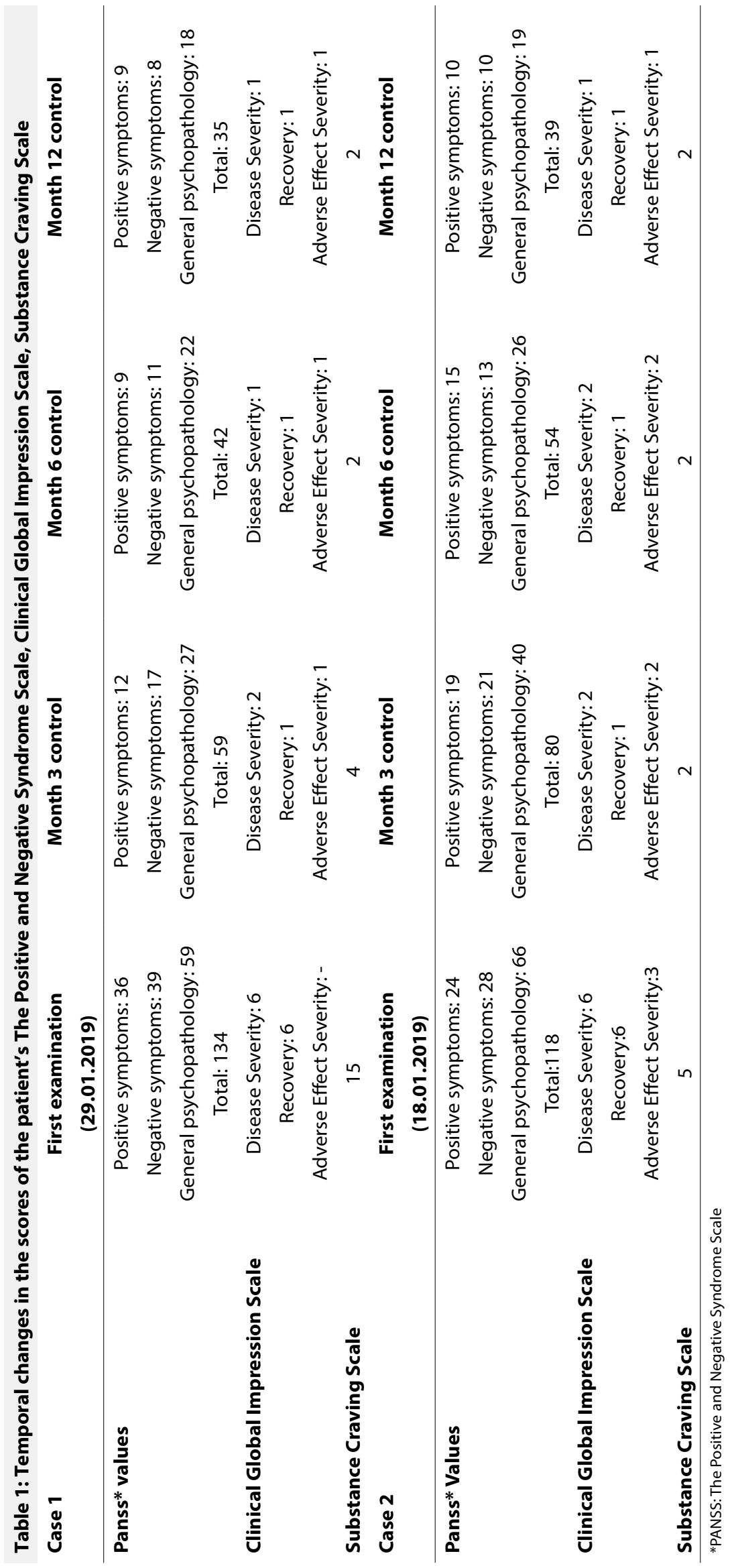

beyond ideation. Several psychotropics were prescribed at different times by different psychiatrists, including alprazolam, paliperidone, lamotrigine, and risperidone, but he did not continue these medications. He was then followed up with a drug regimen, which included haloperidol $15 \mathrm{mg} /$ day, olanzapine $20 \mathrm{mg} /$ day, buspirone $10 \mathrm{mg} /$ day, biperiden $4 \mathrm{mg} /$ day. $\mathrm{He}$ has been using these drugs for the last three years, despite no significant clinical improvement. He has been smoking a pack of cigarettes every day for ten years, and he did not use alcohol. He started substance use nine years ago, initially with cannabinoid, which he continued to take almost every day for the next five years. Additionally, he used ecstasy several times a week and synthetic cannabinoid several times a month. His medical history revealed no other disease. On his mental state examination, he had blunted affect and poverty of speech in addition to persecutory delusions and auditory hallucinations into which he had a partial insight. No substance was detected in urine toxicology, and his routine biochemistry panel (i.e., hemogram, renal function tests, LFT) was within a normal range. The patient was diagnosed with a psychotic disorder specified with a past history of profound substance (cannabis, synthetic cannabinoids and ecstasy) use disorder. Thus, he was taken LAIAM treatment. After administering the second dose of LAIAM, haloperidol was tapered off, whereas other drugs were continued. After 1- year of follow-up, in which no substance use was confirmed by urine toxicology, the patient recovered from his psychotic symptoms and substance craving (Table 1) without experiencing any side effects. His functionality improved, and he started an addiction program. 


\section{DISCUSSION}

We presented two cases treated with LAIAM over a period of 1 year, long enough to evaluate the efficacy, safety and tolerability of this drug. The first case was diagnosed with SIPD, and the second case was diagnosed with psychotic disorder specified with past history of profound substance use disorder. We found that psychotic symptoms improved to a great extent in both of the patients, as evidenced by clinical assessment scales (Table 1). Additionally, the patients were free of craving and could avoid substance use. Thus, after one year of treatment, LAIAM proved to be effective and safe for these two patients, allowing them to return to their premorbid functioning level.

The clinical outcome observed in these patients seems to be consistent with research findings showing the efficacy of aripiprazole in comorbid schizophrenia and substance use disorder. Indeed, oral aripiprazole treatment has been shown to reduce cocaine craving in schizophrenia accompanied by substance use disorder $(9,10)$ and cocaine use disorder (11). Studies conducted with LAIAM also show its efficacy in this patient group. One such study is by Montemitro et al. (12), who reported a high rate of clinical improvement and functional recovery in 18 patients (67\% of 12 patients) with comorbid psychosis and substance use disorder. In another study, LAIAM treatment effectively reduced substance use symptoms in schizophrenia patients with concomitant amphetamine use (13). In a larger sample of schizophrenia patients with comorbid substance use disorder (i.e., 50 patients using LAIAM and 51 patients using long-acting paliperidone depot injection), a significant improvement in both psychotic symptoms and substance craving was observed. They also reported an increase in their quality of life (14). Aripiprazole, like other atypical antipsychotics drugs, has positive effects on a wide variety of cognitive functions, including attention, motor skills, verbal memory, and verbal fluency. Its unique partial agonistic effect on D2 and D3 receptors increases the efficiency of the reward period and reduces depressive symptoms. It is postulated that aripiprazole, with its agonistic property at D3 receptors predominantly found in the mesolimbic pathway (reward system), is implicated in craving because it produces a hyperdopaminergic state. Finally, the low rate of extrapyramidal side effects with aripiprazole is a significant advantage $(15,16)$.

One of the limitations of this case report is that both patients are on an addiction program, as it might also reduce craving. Another limitation is that the two cases are not enough to evaluate the efficacy, safety, and tolerability of any medication, and randomized controlled clinical studies are required. We did not use a scale for side effects that make it difficult to objectively evaluate the side effects of LAIAM, and nor did we use a clinical tool such as SCID-5 for differential diagnosis, both of which may also be considered as limitations.

Taken together, all these suggest that aripiprazolemaybe an effective and safe treatment option, especially in its long-acting form (LAIAM), in SIPD and patients with dual diagnosis of psychotic disorder and substance use disorder. Although we did not evaluate its cognitive effects in our cases, the outcome was favorable considering the clinical improvement, the absence of side effects, and functional recovery in both of these patients. Given the scarcity of evidence regarding the use of longacting antipsychotics, including LAIAM, in the treatment of SIPD, we think that our observations in these two cases could contribute to the current knowledge and help practitioners in their clinical practice. However, it is worthy of note that studies with longer follow-up and larger samples are needed, including the evaluation of cognitive functions.

\begin{tabular}{|c|c|c|}
\hline \multicolumn{2}{|c|}{ Contribution Categories } & \multirow{2}{*}{$\begin{array}{l}\text { Author Initials } \\
\text { A.E., B.K., M.S.B., B.C., M.M.K. }\end{array}$} \\
\hline \multirow{4}{*}{ Category 1} & Concept/Design & \\
\hline & Literature review & A.E., B.K., M.S.B., B.C., M.M.K. \\
\hline & Data analysis/Interpretation & A.E., B.K., M.S.B., B.C., M.M.K. \\
\hline & Case follow-up (if applicable) & A.E., B.K., M.S.B., B.C., M.M.K. \\
\hline \multirow{2}{*}{ Category 2} & Drafting manuscript & A.E., B.K., M.S.B., B.C., M.M.K. \\
\hline & Critical revision of manuscript & A.E., B.K., M.S.B., B.C., M.M.K. \\
\hline Category 3 & Final approval and accountability & A.E., B.K., M.S.B., B.C., M.M.K. \\
\hline \multirow{2}{*}{ Other } & Technical or material support & A.E., B.K., M.S.B., B.C., M.M.K. \\
\hline & Supervision & A.E., B.K., M.S.B., B.C., M.M.K. \\
\hline
\end{tabular}

Informed Consent: Written informed consent was obtained from both patients.

Peer-review: Externally peer-reviewed.

Conflict of Interest: None declared.

Financial Disclosure: No financial support has been received from any institution or person for this study.

\section{REFERENCES}

1. Martin DA, Marona-Lewicka D, Nichols DE, Nichols CD. Chronic LSD alters gene expression profiles in the mPFC relevant to schizophrenia. Neuropharmacology 2014; 83:1-8.

2. Tenn CC, Fletcher PJ, Kapur S. Amphetamine-sensitized animals show a sensorimotor gating and neurochemical abnormality similar to that of schizophrenia. Schizophr Res 2003; 64:103-114. 
3. Manrique-Garcia E, Zammit S, Dalman C, Hemmingsson T, Andreasson S, Allebeck P. Cannabis, schizophrenia and other non-affective psychoses: 35 years of follow-up of a populationbased cohort. Psychol Med 2012; 42:1321-1328.

4. American Psychiatric Association. Diagnostic And Statistical Manual Of Mental Disorders. 5th ed. Arlington, VA: American Psychiatric Publishing, 2013.

5. Wilson L, Szigeti A, Kearney A, Clarke M. Clinical characteristics of primary psychotic disorders with concurrent substance abuse and substance-induced psychotic disorders: A systematic review. Schizophr Res 2018; 197:78-86.

6. Nosé M, Barbui C, Tansella M. How often do patients with psychosis fail to adhere to treatment programmes? A systematic review. Psychol Med 2003; 33:1149-1160.

7. Marcus SC, Zummo J, Pettit AR, Stoddard J, Doshi JA. Antipsychotic adherence and rehospitalization in schizophrenia patients receiving oral versus long-acting injectable antipsychotics following hospital discharge. J Manag Care Spec Pharm 2015; 21:754-768.

8. Chue P, Chue J. A review of aripiprazole long-acting injection. Curr Med Res Opin 2016; 32:441-452.

9. Beresford TP, Clapp L, Martin B, Wiberg JL, Alfers J, Beresford HF. Aripiprazole in schizophrenia with cocaine dependence: a pilot study. J Clin Psychopharmacol 2005; 25:363-366.

10. Beresford T, Buchanan J, Thumm EB, Emrick C, Weitzenkamp $\mathrm{D}$, Ronan PJ. Late reduction of cocaine cravings in a randomized, double-blind trial of aripiprazole vs perphenazine in schizophrenia and comorbid cocaine dependence. J Clin Psychopharmacol 2017; 37:657-663.

11. Meini M, Moncini M, Cecconi D, Cellesi V, Biasci L, Simoni G, et al. Safety, tolerability, and self-rated effects of aripiprazole and ropinirole treatment for cocaine dependence: a pilot study. Am J Addict 2011; 20:179-180

12. Montemitro C, Baroni G, Cantelmi V, Tittozzi B, Pettoruso $M$, Di Nicola $M$, et al. Long-acting injectable aripiprazole as treatment for psychiatric patients comorbid with substancerelated and addictive disorder: efficacy and safety. Eur Neuropsychopharmacol 2017; 27:1072.

13. Chen SF, Shen YC. Long-acting injectable aripiprazole for a schizophrenic patient concomitant with stimulant use disorder. J Clin Psychopharmacol 2019; 39:508-509.

14. Cuomo I, Kotzalidis GD, de Persis S, Piacentino D, Perrini F, Amici E, et al. Head-to-head comparison of 1-year aripiprazole long-acting injectable (LAI) versus paliperidone LAI in comorbid psychosis and substance use disorder: impact on clinical status, substance craving, and quality of life. Neuropsychiatr Dis Treat 2018; 14:1645-1656.

15. Burris KD, Molski TF, Xu C, Ryan E, Tottori K, Kikuchi T, et al. Aripiprazole, a novel antipsychotic, is a high-affinity partial agonist at human dopamine D2 receptors. J Pharmacol Exp Ther 2002; 302:381-389.

16. Tadori Y, Forbes RA, McQuade RD, Kikuchi T. Characterization of aripiprazole partial agonist activity at human dopamine D3 receptors. Eur J Pharmacol 2008; 597:27-33. 